\title{
Silencing of cyclooxygenase- 2 inhibits the growth, invasion and migration of ovarian cancer cells
}

\author{
YANG LIN, MANHUA CUI, TIANMIN XU, WEI YU and LIHUI ZHANG
}

Department of Gynaecology and Obstetrics, The Second Hospital of Jilin University, Changchun, Jilin 130041, P.R. China

Received August 26, 2013; Accepted March 10, 2014

DOI: $10.3892 / \mathrm{mmr} .2014 .2131$

\begin{abstract}
The present study aimed to investigate the effect of downregulating cyclooxygenase-2 (COX-2) expression on the growth of human ovarian cancer cells. The COX-2-specific small interfering RNA (siRNA) plasmid vector was constructed and then transfected into ovarian cancer cells. The expression of COX-2 mRNA and protein was detected by quantitative polymerase chain reaction and western blot analysis, respectively. Cell proliferation, apoptosis, cell cycle distribution and cell migration were assessed following knockdown of COX-2 by RNA interference (RNAi). Western blot analysis was used to identify differentially expressed angiogenesis- and cell cycle-associated proteins in cells with silenced COX-2. The expression levels of COX-2 in ovarian cancer cells transfected with siRNA were decreased, leading to a significant inhibition of ovarian cancer cell proliferation, migration and invasion. Western blot analysis revealed that silencing of COX-2 may inhibit vascular endothelial growth factor, matrix metalloproteinase (MMP)-2 and MMP-9 protein expression. In conclusion, the present study demonstrated that RNAi can effectively silence COX-2 gene expression and inhibit the growth of ovarian cancer cells, which indicates that there is a potential of targeting COX-2 as a novel gene therapy approach for the treatment of ovarian cancer.
\end{abstract}

\section{Introduction}

Ovarian cancer is one of the most common types of fatal tumors of the female reproductive tract and also a major cause of mortality resulting from gynecological malignancies (1). Epithelial ovarian cancer is the predominant form among ovarian cancers and accounts for $90 \%$ of incidences; the majority of mortalities are due to this malignancy (2).

Correspondence to: Professor Lihui Zhang, Department of Gynaecology and Obstetrics, The Second Hospital of Jilin University, Ziqing Street, Changchun, Jilin 130041, P.R. China E-mail: zhanglihui538@163.com

Key words: RNA interference, cyclooxygenase-2, cell proliferation, ovarian cancer
There is a lack of effective screening and early detection strategies; therefore, the majority of females are diagnosed with advanced-stage metastatic cancer for which surgical and pharmaceutical treatment options are significantly less effective $(3,4)$. Standard treatment options include debulking followed by chemotherapy with platinum agents. Although there is a good response to primary surgery and chemotherapy treatments, the recurrence rates are high $(>60 \%)$ and salvage therapies available are not curative (5-8). Therefore, it is important to understand the molecular mechanisms underlying this disease in order to develop novel treatment strategies to improve the clinical outcomes for these patients.

Cyclooxygenase-2 (COX-2) is inducible by inflammatory stimuli, including cytokines, growth factors and tumor promoters, and is upregulated in a variety of malignancies. COX-2 upregulation favors the growth of malignant cells by stimulating proliferation and angiogenesis $(9,10)$. A large number of previous studies demonstrated that COX-2 is overexpressed in ovarian cancer (11-13). Furthermore, Arico et al (14) found that COX-2 can induce angiogenesis via vascular endothelial growth factor (VEGF) and prostaglandin production and can also inhibit apoptosis by inducing the anti-apoptotic factor B-cell lymphoma 2 as well as activating anti-apoptotic signaling through $\mathrm{Akt} /$ protein kinase $\mathrm{B}$ (one of the serine/threonine kinases). These results suggest that COX-2 has a significant role in the generation and progression of solid tumors and the inhibition of COX-2 may inhibit the growth of a variety of solid malignancies. Therefore, downregulation of COX-2 in cancer cells may prove useful in improving clinical outcomes in cancer patients.

RNA interference (RNAi) is a powerful method for gene inactivation (15-17) and cancer gene therapy (18). The basic mechanism of RNAi starts with a long double-stranded RNA that is processed into small interfering RNAs (siRNAs) of $\sim 21 \mathrm{nt}(19,20)$. The advantage of RNAi technology is that it can be used to target a large number of different genes, which are involved in a number of distinct cellular pathways. The technology of RNA silencing is poised to have a major impact on the treatment of human disease, particularly cancer (19).

The aim of the present study was to investigate the role of COX-2 in the growth of human ovarian cancer cells. The effect of RNAi-induced COX-2 suppression on the proliferation, invasion and migration of ovarian cancer cells was also evaluated. 


\section{Materials and methods}

Cell culture. SKOV3 cells were obtained from the American Type Culture Collection (Manassas, VA, USA) and cultured in Modified McCoy's 5A Medium (Sigma, St. Louis, MO, USA) supplemented with $10 \%$ fetal bovine serum (FBS) (Gibco-BRL, Carlsbad, CA, USA). The cells were maintained in a humidified $37^{\circ} \mathrm{C}$ incubator with $5 \% \mathrm{CO}_{2}$.

siRNA design and conduct. In total, three different siRNAs were designed against COX-2 mRNA as suggested by the method used in a study by Elbashir et al (21). The targeting sequences corresponding to the siRNAs against COX-2 (GeneBank accession no. NM_000963.2) were as follows: Bases 290-310 (siRNA-1, 5'-AAACTGCTCAACACCGGAATT-3'), bases 456-477 ('siRNA-2,5'-TCACATTTGATTGACAGTCCA-3') and 517-538 (siRNA-3, 5'-CCTTCTCTAACCTCTCCTATT-3'). The interference vector pGenesil-COX-2 was constructed using the pGenesil-1 vector. In brief, three pairs of oligonucleotide fragments were designed, synthesized and annealed. pGenesil-1, which consisted of human U6 short hairpin RNA (shRNA) promoter, was used to generate a series of siRNA expression vectors by inserting three pairs of annealed oligonucleotides. The recombinant plasmid vectors pGenesil-1-COX-2(1), pGenesil-1-COX-2(2) and pGenesil-1-COX-2(3) were repeatedly excised and ligated successively. Thus, the tandem recombinant vector pGenesil-1-COX-2(1+2+3) was constructed and called pGenesil-1-COX-2. All the sequences inserted were verified by DNA sequencing. Plasmid pGenesil-1-KB was used to serve as a control for the empty vector. All the siRNA sequences were checked in terms of their specificity using the Basic Local Alignment Search Tool (National Library of Medicine, Bethesda, MD, USA) database and did not exhibit any homology to other human genes.

The SKOV3 cells were randomly divided into three groups: Untransfected control group, empty vector transfected vector group that served as a blank control, and the pGenesil-1-COX-2 siRNA expression plasmid transfected siRNA group. In total, $5 \times 10^{5}$ cells were seeded in six-well plates and grown overnight prior to transfection. All the plasmids were transiently transfected into SKOV3 cells by Lipofectamine ${ }^{\mathrm{TM}} 2000$ (Invitrogen $^{-1}$ Life Technologies, Carlsbad, CA, USA) according to the manufacturer's instructions and incubated in serum-starved media at $37^{\circ} \mathrm{C}$ in $5 \% \mathrm{CO}_{2}$. At $8 \mathrm{~h}$ post-transfection, the medium was replaced with fresh complete medium containing $10 \%$ FBS. The cells were harvested $48 \mathrm{~h}$ after transfection and used for the evaluation of COX-2 expression. The transfection efficiency was monitored by measuring the percentage of fluorescent cells among a total of 1,000 cells using fluorescence microscopy.

RNA isolation and quantitative polymerase chain reaction ( $q P C R$ ). To evaluate COX-2 mRNA expression, the cells were harvested following transfection. The total RNA was extracted from the cells using TRIzol reagent (Invitrogen Life Technologies) for reverse transcription. The RNA was transcribed to cDNA using the Superscript First-Strand Synthesis kit (Takara, Dalian, China) following the manufacturer's instructions. qPCR assays were performed using SYBR-Green Real-Time PCR Master Mix
(Toyobo, Osaka, Japan) and RT-PCR amplification equipment (7300 Real-Time PCR System; Applied Biosystems, Foster City, CA, USA) using specific primers: COX-2 sense, 5'-CCCTTGGGTGTCAAAGGTAAA-3' and antisense, 5'-AAACTGATGCGTGAAGTGCTG-3'; and $\beta$-actin sense, 5'-GCGAGCACAGAGCCTCGCCTTTG-3' and antisense, 5'-GATGCCGTGCTCGATGGGGTAC-3'. The PCR conditions were as follows: Pre-denaturation at $95^{\circ} \mathrm{C}$ for $2 \mathrm{~min}$, followed by 40 cycles of denaturation at $95^{\circ} \mathrm{C}$ for $10 \mathrm{sec}$ and annealing/extension at $58^{\circ} \mathrm{C}$ for $20 \mathrm{sec}$. The amplification specificity was checked by melting curve analysis. The expression of the genes of interest was determined by normalization of the threshold cycle $(\mathrm{Ct})$ of these genes to that of the $\beta$-actin control.

Western blot analysis. The cells were harvested and lysed in Triton X-100 in Hepes buffer [150 mM NaCl, 50 mM Hepes, $1.5 \mathrm{mM} \mathrm{MgCl}_{2}, 1 \%$ Triton X-100, $0.1 \%$ sodium dodecyl sulfate (SDS) and a protease inhibitor cocktail (Sigma)]. Western blot analysis was performed using conventional protocols. Briefly, the protein concentration of the extracts was determined using a bicinchoninic acid kit (Sigma) with bovine serum albumin used as the standard. The total protein samples $(40 \mu \mathrm{g})$ were separated by $8 \%$ SDS-PAGE, proteins were transferred to nitrocellulose membranes (Millipore, Bedford, MA, USA), immunoblotted with specific primary antibodies and incubated with corresponding horseradish peroxidase (HRP)-conjugated secondary antibody. The other primary antibodies used in the western blots were as follows: Antibodies against COX-2, $\beta$-actin, VEGF (Santa Cruz Biotechnology, Inc., Santa Cruz, CA, USA); MMP-9 and MMP-2 (Sigma Aldrich, St. Louis, MO, USA); secondary antibodies used for immunodetection were as follows: HRP-conjugated goat anti-mouse immunoglobulin (Ig) $\mathrm{G}$ and goat anti-rabbit IgG (Amersham Biosciences, Uppsala, Sweden). All the immunoblots were visualized by enhanced chemiluminescence (Pierce Biotechnology, Inc., Rockford, IL, USA). All the assays using COX-2 knockdown SKVO3 cells were performed after the third day of siRNA transfection.

Cell viability assay. At 24, 48 and 72 h post-transfection with COX-2 siRNAs, the cells were seeded in quadruplicate into 96-well plates (5,000 cells/well in $100 \mathrm{ml}$ of medium). At the indicated times, the cells were incubated with $1 \mathrm{mg} / \mathrm{ml} \mathrm{MTT}$ in normal culture medium for $6 \mathrm{~h}$ at $37^{\circ} \mathrm{C}$. The medium was then aspirated and the formazan was dissolved in $200 \mathrm{ml}$ dimethyl sulfoxide. The absorbance was measured at $570 \mathrm{~nm}$ by a STAKMAX ${ }^{\mathrm{TM}}$ microplate reader (Molecular Devices, Sunnyvale, CA, USA) and the experiments were repeated five times.

Cell cycle analysis. The SKVO3 cells were treated with siRNA for $24 \mathrm{~h}$ in Dulbecco's modified Eagle's medium (DMEM) containing 5\% FBS. All the cells were collected, and $1 \times 10^{6}$ cells were centrifuged, resuspended in ice-cold $70 \%$ ethanol and stored at $-20^{\circ} \mathrm{C}$ until further analysis. Washed cells were stained by $0.1 \%$ Triton $\mathrm{X}-100$ in $0.01 \mathrm{M}$ phosphate-buffered saline ( $\mathrm{pH} 7.2$ ) with $50 \mu \mathrm{g} / \mathrm{ml}$ propidium iodide (Sigma-Aldrich) and $1 \mathrm{mg} / \mathrm{ml}$ RNase A (Invitrogen), and incubated at $37^{\circ} \mathrm{C}$ for $30 \mathrm{~min}$ in the dark. Samples of the 
A

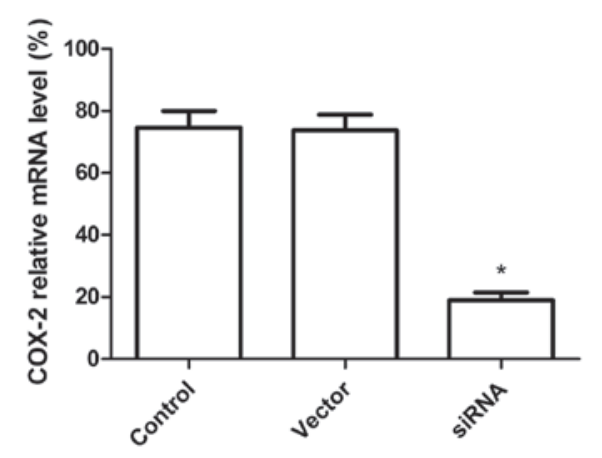

B

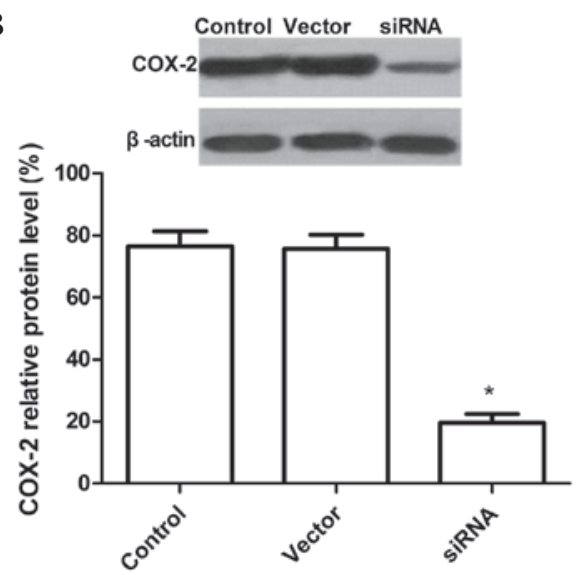

Figure 1. Silencing of COX-2 decreases COX-2 expression in SKVO3 tumor cells. (A) Quantitative polymerase chain reaction analysis of COX-2 after RNAi silencing. (B) Western blot analysis of COX-2 after RNAi silencing. ${ }^{*} \mathrm{P}<0.05$ and ${ }^{* *} \mathrm{P}<0.01$ vs. the control. RNAi, RNA interference; COX-2, cyclooxygenase-2.

cells were then analyzed for their DNA content using FACScan flow cytometry (Beckman, Miami, FL, USA), and cell cycle phase distributions were analyzed by the Cell Quest acquisition software (BD Biosciences, Franklin Lanes, NJ, USA). All experiments were performed in duplicate and repeated twice.

Cell migration assay. The migration assay was performed using a 12-well Boyden Chamber (Neuro Probe, Inc., Gaithersburg, MD, USA) with an $8 \mu \mathrm{m}$ pore size. In total, $\sim 1 \times 10^{5}$ cells were seeded into upper wells of the Boyden Chamber and incubated for $6 \mathrm{~h}$ at $37^{\circ} \mathrm{C}$ in medium containing $1 \%$ FBS. DMEM with $10 \%$ FBS was used as a chemoattractant in the bottom wells. The cells that did not migrate through the pores of the Boyden Chamber were manually removed with a rubber swab. The cells that migrated to the lower side of the membrane were stained with hematoxylin and eosin and photographed using an inverted microscope.

Transwell invasion assay. The invasiveness of SKVO3 cancer cells was assessed using 24-well Transwell plates (Corning, Lowell, MA, USA). In brief, $2 \times 10^{5}$ cells in DMEM with $0.5 \%$ FBS were added to the upper chamber containing a 8 -mm pore polycarbonate coated with $1 \mathrm{mg} / \mathrm{ml}$ Matrigel; the lower chamber was filled with media containing 5\% FBS. Subsequent to $16 \mathrm{~h}$ incubation, the upper surface of the membrane was scrubbed with a cotton-tipped swab. The invading cells on the lower surface of the membrane were fixed and stained with $0.5 \%$ crystal violet dye. A total of five random fields per membrane were photographed at magnification, $x 40$ for calculating the cell number using an inverted phase-contrast microscope (Leica, Solms, Germany). In addition, cells were quantified by measuring the absorbance of dye extracts at $570 \mathrm{~nm}$ in $100 \mathrm{ml}$ of Sorenson's solution ( $9 \mathrm{mg}$ trisodium citrate, $305 \mathrm{ml}$ distilled water, $195 \mathrm{ml} 0.1 \mathrm{~N} \mathrm{HCl}$ and $500 \mathrm{ml}$ 90\% ethanol). All experiments were performed in triplicate and repeated four times.

Determination of prostaglandin E2 (PGE2) synthesis by ELISA. PGE2 synthesis was determined according to methods that were previously described (22). In brief, the SKVO3 cells were grown in 12-well plates overnight. The culture media of the cells were changed to new DMEM for $30 \mathrm{~min}$ prior to harvesting of culture media and then these culture media were centrifuged to remove cell debris. Cell-free culture media were collected at indicated time periods, then PGE2 levels were determined using a Human PGE2 ELISA kit according to the manufacturer's instructions (Cayman Chemical, Ann Arbor, MI, USA) and an ELISA reader ( $\mu$ Quant; Biotek Instruments, Inc., Winooski, VT, USA).

Statistical analysis. The values were expressed as the mean \pm standard deviation. The data were analyzed by one-way analysis of variance and $\mathrm{P}<0.05$ was considered to be statistically significant.

\section{Results}

Downregulation of COX-2 $m R N A$ and protein expression levels by $C O X-2-s i R N A$. A recombinant vector was designed and constructed, which expresses three siRNAs targeting the COX-2 gene in tandem, and transfected it into SKOV3 cells. Subsequent to $72 \mathrm{~h}$ transfection, the cells were harvested and the COX-2 mRNA levels were analyzed by qPCR. COX-2 expression was significantly downregulated in SKOV3 cells transfected with siRNA compared with the cells transfected with the control vector and untransfected cells $(\mathrm{P}<0.01)$. There was no significant difference between the untransfected and empty vector groups $(\mathrm{P}>0.05)$ (Fig. 1A). The results indicated that most of the COX-2 mRNA was degraded by COX-2 siRNA in the SKOV3 cells.

The effect of COX-2 siRNA treatment on protein expression was assessed by western blot analysis. As shown in Fig. 1B, there was no difference between the untransfected and empty vector group $(\mathrm{P}>0.05)$, while the band density clearly decreased in the COX-2 siRNA group as compared with the untransfected and empty vector group. These results demonstrated that siRNA targeting COX-2 significantly silenced COX-2 protein expression in SKOV3 ovarian cancer cells $(\mathrm{P}<0.01)$.

COX-2 silencing affects $\mathrm{SKVO} 3$ cell proliferation and the cell cycle. To elucidate the effects of the siRNA on SKOV3 ovarian cancer cell proliferation, the MTT assay was used and cell proliferation was determined by counting the number of viable cells. SKOV3 ovarian cancer cells transfected with 
A

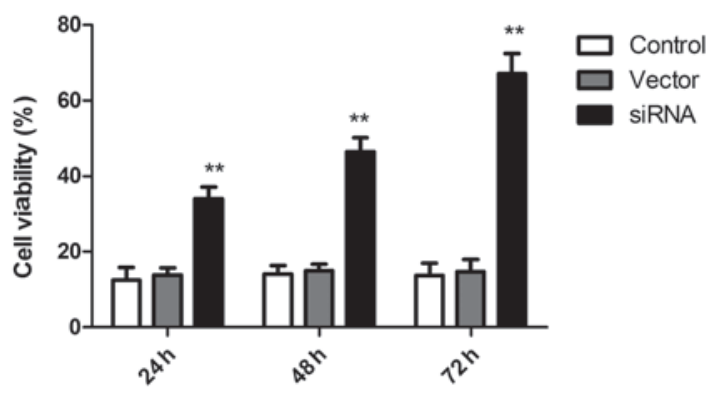

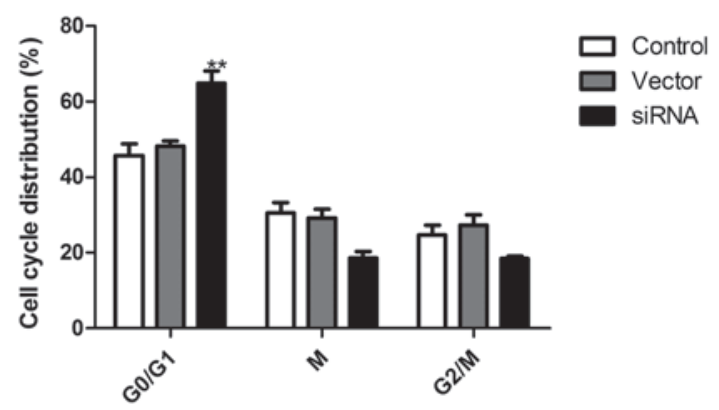

Figure 2. Silencing of COX-2 inhibits cell proliferation and the cell cycle in vitro. (A) Downregulation of COX-2 by siRNA significantly suppressed the proliferation and (B) induced G0/G1 phase arrest of SKVO3 cells. " $\mathrm{P}<0.05$ and ${ }^{* *} \mathrm{P}<0.01$ vs. the control. siRNA, small interfering RNA; COX-2, cyclooxygenase-2.

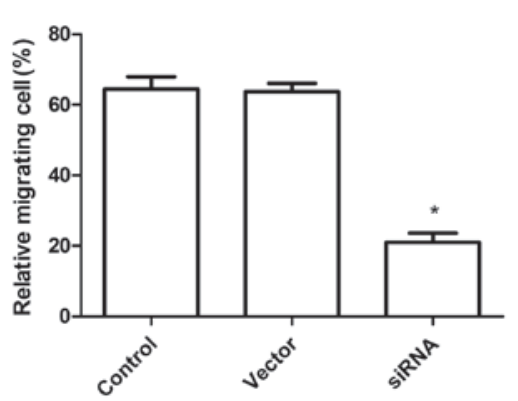

B

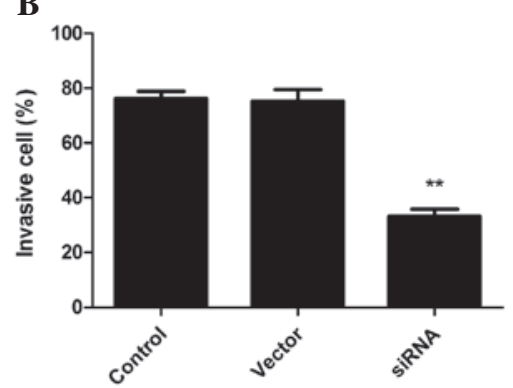

Figure 3. Silencing of COX-2 affects cell migration and invasion in vitro. Downregulation of the COX-2 gene by siRNA caused significantly suppressed (A) cell migration and (B) cell invasion of SKVO3 cells. ${ }^{*} \mathrm{P}<0.05$ and ${ }^{* *} \mathrm{P}<0.01$ vs. the control. siRNA, small interfering RNA; COX-2, cyclooxygenase-2.

A

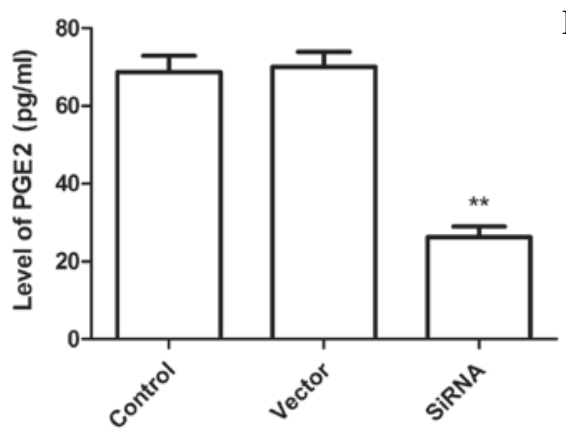

B

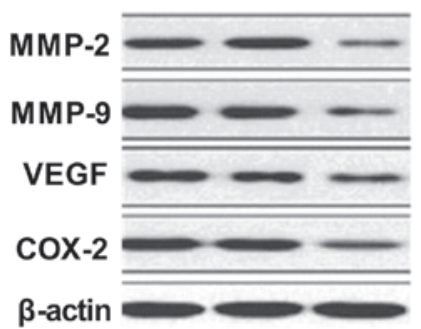

Figure 4. Silencing of COX-2 affects PGE2 expression by ELISA, and related protein expression by western blot analysis. (A) PGE2 levels were significantly decreased after silencing COX-2 as compared with the control cells. " $\mathrm{P}<0.05$ and ${ }^{* *} \mathrm{P}<0.01$ vs. the control. (B) Protein expression levels of VEGF, MMP-9 and MMP-2 were detected by western blot analysis after COX-2 silencing (left lane, control; middle lane, vector; right lane, siRNA group). PGE2, prostaglandin E2; VEGF, vascular endothelial growth factor; MMP, metalloproteinase; COX-2, cyclooxygenase-2.

COX-2-siRNA proliferated at a much lower rate compared with the control cells at 24,48 and $72 \mathrm{~h}$ post-transfection. Compared with the untransfected cells, the proliferation properties of SKOV3 cells were significantly inhibited (Fig. 2A). These data demonstrate that the inhibition of COX-2 by RNAi can inhibit the proliferation of SKOV3 cells.

In addition, to determine the effects of SKVO3 cell cycle progression following $\mathrm{COX}-2$ silencing, flow cytometry was performed in the present study. In the siRNA therapy group, the percentage of cells in G0/G1 phase was significantly increased as compared with the scrambled-treated and control cells. These results indicated that COX-2 silencing can induce cell cycle arrest in G0/G1 phase in $\mathrm{SKVO} 3$ cells (Fig. 2B).
COX-2 silencing inhibits SKVO3 cell invasion and cell migration. To analyze whether siRNA affects SKVO3 cell migration, migration assays were performed using Boyden chambers. RNAi-mediated COX-2 silencing significantly inhibited SKVO3 cell migration as compared with the control (untransfected) and negative control-transfected (Vector) groups (Fig. 3A). On the other hand, COX-2 knockdown in SKVO3 cells markedly inhibited invasion in vitro as compared with the vector-treated and control groups (Fig. 3B). The control cells and vector cells remained invasive and no statistically significant differences were observed.

Silencing of COX-2 decreases levels of PGE2 and other proteins in SKVO3 cells. PGE2 levels in SKVO3 cells were 
determined by ELISA analysis. As shown in Fig. 4A, the PGE2 levels in the siRNA therapy groups were significantly lower compared with those in the untransfected and empty vector groups. In addition, VEGF protein expression levels were determined following COX-2 silencing. It was found that COX-2 silencing was found to significantly inhibit VEGF expression in the SKVO3 tumor cells compared with the control and empty vector cells (Fig. 4B).

In order to investigate the mechanisms involved in the inhibition of the invasion and migration ability by downregulation of COX-2 silencing of SKVO3 cells, western blot analysis was performed to evaluate the activity of MMP-2 and MMP-9. COX-2 silencing caused a significant decrease in MMP-9 and MMP-2 levels compared with the control cells as determined by western blot analysis (Fig. 4B).

\section{Discussion}

RNAi is a fundamental cellular mechanism used for silencing gene expression (16,23-25). RNAi has been widely used in cancer therapy to silence the expression of oncogenes and growth factors or their receptors, which may result in inhibition of the cell cycle, cell proliferation and tumor angiogenesis as well as induction of cell apoptosis (26-28). To date, RNAi has been regarded as an effective and useful approach for therapeutic applications of cancer $(26,39)$. In the present study, RNAi strategies were used to reduce the expression of COX-2 in the ovarian cancer cell line SKOV3 and to evaluate the role of COX-2 in SKOV3 cells. A total of three siRNA sequences were designed to target the COX-2 coding region and inserted into the pGenesil-1 vector in tandem to efficiently suppress the expression of COX-2. The present study demonstrated that this vector was efficiently silenced COX-2. Downregulation of COX-2 reduced proliferation, cell cycle, cell migration and invasion in SKOV3 cells. The results were consistent with those of previous studies revealing that the number of viable cells was significantly decreased following transfection with COX-2 siRNA $(28,30,31)$.

COX-2 is known to be involved in multiple pathophysiological processes, including inflammation and tumorigenesis $(32,33)$. COX-2 is undetectable in numerous normal tissues, yet it is commonly overexpressed in various human cancers, including ovarian cancer (14), with its downstream product being PGE2, which is linked to more aggressive behavior of tumors and thus contributing to ovarian cancer progression $(34,35)$. PGE2 is a significant mediator in tumor-promoting inflammation (36). Additionally, PGE2 promotes tumor cell proliferation, induces VEGF upregulation and inhibits tumor cell apoptosis as well as immune function (37). The present study revealed that downregulation of the COX-2 gene by silencing decreased the levels of PGE2 and VEGF (Fig. 4B) and inhibit tumor cell proliferation and the cell cycle. These results implied that the major mechanism of COX-2 in stimulating tumorigenesis is via its product PGE2.

The poor prognosis of ovarian cancer is mainly due to dissemination caused by the aggressive migration activity of the cancer cells (38). In the present study, siRNA-mediated downregulation of COX-2 expression in human ovarian cancer cells lead to a significant decrease in SKOV3 cell invasion and migration. These results were consistent with previous studies $(28,30,31)$, demonstrating that COX-2 mediates the invasive and metastatic potential of ovarian cancer cells.

In conclusion, the vector expressing three siRNAs targeting the COX-2 gene in tandem was able to silence the expression of COX-2 in ovarian cancer cells. The COX-2 knockdown not only resulted in a decrease of cell proliferation and inhibition of the cell cycle in ovarian tumor cells, but it also suppressed cell migration and invasion of ovarian cancer cells. These results indicated that COX-2 is a potential therapeutic target for the prevention or treatment of ovarian cancer.

\section{Acknowledgements}

This study was supported by the National Natural Science Foundation of Jilin (no. 83657432).

\section{References}

1. Lin Y, Peng S, Yu H, Teng H and Cui M: RNAi-mediated downregulation of NOB1 suppresses the growth and colony-formation ability of human ovarian cancer cells. Med Oncol 29: 311-317, 2012.

2. Auersperg N, Wong AS, Choi KC, Kang SK and Leung PC: Ovarian surface epithelium: biology, endocrinology, and pathology. Endocr Rev 22: 255-258, 2001.

3. Ziebarth AJ, Landen CN Jr and Alvarez RD: Molecular/genetic therapies in ovarian cancer: future opportunities and challenges. Clin Obstet Gynecol 55: 156-172, 2012.

4. Wang H, Linghu H, Wang J, Che YL, Xiang TX, Tang WX and Yao ZW: The role of Crk/Dock180/Rac1 pathway in the malignant behavior of human ovarian cancer cell SKOV3. Tumour Biol 31: 59-67, 2010.

5. Fader AN and Rose PG: Role of surgery in ovarian carcinoma. J Clin Oncol 25: 2873-2883, 2007.

6. Salzberg M, Thurlimann B, Bonnefois H, Fink D, Rochlitz C, Von Moos R and Senn H: Current concepts of treatment strategies in advanced or recurrent ovarian cancer. Oncology 68: 293-298, 2005.

7. McGuire WP and Ozols RF: Chemotherapy of advanced ovarian cancer. Semin Oncol 25: 340-348, 1998.

8. Fung-Kee-Fung M, Oliver T, Elit L, Oza A, Hirte HW and Bryson P: Optimal chemotherapy treatment for women with recurrent ovarian cancer. Curr Oncol 14: 195-208, 2007.

9. Dempke W, Rie C, Grothey A and Schmoll HJ: Cyclooxygenase-2: a novel target for cancer chemotherapy? J Cancer Res Clin Oncol 127: 411-417, 2001.

10. Williams CS, Mann M and DuBois RN: The role of cyclooxygenases in inflammation, cancer, and development. Oncogene 18: 7908-7916, 1999.

11. Li S, Miner K, Fannin R, Carl Barrett $\mathrm{J}$ and Davis BJ: Cyclooxygenase- 1 and 2 in normal and malignant human ovarian epithelium. Gynecol Oncol 92: 622-627, 2004.

12. Denkert C, Köbel M, Pest S, Koch I, Berger S, Schwabe M, Siegert A, Reles A, Klosterhalfen B and Hauptmann S: Expression of cyclooxygenase-2 is an independent prognostic factor in human ovarian carcinoma. Am J Pathol 160: 893-903, 2002.

13. Erkinheimo TL, Lassus H, Finne P, van Rees BP, Leminen A, Ylikorkala O, Haglund C, Butzow R and Ristimäki A: Elevated cyclooxygenase-2 expression is associated with altered expression of p53 and SMAD4, amplification of HER-2/neu, and poor outcome in serous ovarian carcinoma. Clin Cancer Res 10: 538-545, 2004.

14. Arico S, Pattingre S, Bauvy C, Gane P, Barbat A, Codogno P and Ogier-Denis E: Celecoxib induces apoptosis by inhibiting 3 -phosphoinositide-dependent protein kinase-1 activity in the human colon cancer HT-29 cell line. J Biol Chem 277: 27613-27621, 2002.

15. Cerutti H: RNA interference: traveling in the cell and gaining functions? Trends Genet 19: 39-46, 2003. 
16. Fire A, Xu S, Montgomery MK, Kostas SA, Driver SE and Mello CC: Potent and specific genetic interference by double-stranded RNA in Caenorhabditis elegans. Nature 391: 806-811, 1998.

17. Hannon GL and Rossi JJ: Unlocking the potential of the human genome with RNA interference. Nature 431: 371-378, 2004.

18. Izquierdo M: Short interfering RNAs as a tool for cancer gene therapy. Cancer Gene Ther 12: 217-227, 2005.

19. Elbashir SM, Harborth J, Lendeckel W, Yalcin A, Weber K and Tuschl T: Duplexes of 21-nucleotide RNAs mediate RNA interference in cultured mammalian cells. Nature 411: 494-498, 2001.

20. Caplen NJ: Gene therapy progress and prospects. Downregulating gene expression: the impact of RNA interference. Gene Ther 11: 1241-1248, 2004.

21. Elbashir SM, Harborth J, Weber K and Tuschl T: Analysis of gene function in somatic mammalian cells using small interfering RNAs. Methods 26: 199-213, 2002.

22. Tai MH, Weng CH, Mon DP, Hu CY and Wu MH: Ultraviolet C irradiation induces different expression of cyclooxygenase 2 in NIH 3T3 cells and A431 cells: the roles of COX-2 are different in various cell lines. Int J Mol Sci 13: 4351-4366, 2012.

23. Kim DH, Longo M, Han Y, Lundberg P, Cantin E and Rossi JJ: Interferon induction by siRNAs and ssRNAs synthesized by phage polymerase. Nat Biotechnol 22: 321-325, 2004.

24. Meister G and Tuschi T: Mechanisms of gene silencing by double-stranded RNA. Nature 431: 343-349, 2004.

25. Mello CC and Conte D Jr: Revealing the world of RNA interference. Nature 431: 338-342, 2004.

26. Wang R, Wang X, Lin F, Gao P, Dong K and Zhang HZ: shRNA-targeted cyclooxygenase (COX)-2 inhibits proliferation, reduces invasion and enhances chemosensitivity in laryngeal carcinoma cells. Mol Cell Biochem 317: 179-188, 2008.

27. Chen Q, Pan Q, Cai R and Qian C: Prospects of RNA interference induced by RNA Pol II promoter in cancer therapy. Prog Biochem Biophys 34: 806-815, 2007.

28. Strillacci A, Griffoni C, Spisni E, Manara MC and Tomasi V: RNA interference as a key to knockdown overexpressed cyclooxygenase-2 gene in tumour cells. Br J Cancer 94: 1300-1310, 2006 .
29. Vogler M, Walczak H, Stadel D, Haas TL, Genze F, Jovanovic M, Gschwend JE, Simmet T, Debatin KM and Fulda S: Targeting XIAP bypasses Bcl-2-mediated resistance to TRAIL and cooperates with TRAIL to suppress pancreatic cancer growth in vitro and in vivo. Cancer Res 68: 7956-7965, 2008.

30. Zhang L, Wu YD, Li P, Tu J, Niu YL, Xu CM and Zhang ST: Effects of cyclooxygenase-2 on human esophageal squamous cell carcinoma. World J Gastroenterol 17: 4572-4580, 2011.

31. Denkert C, Fürstenberg A, Daniel PT, Koch I, Köbel M, Weichert W, Siegert A and Hauptmann S: Induction of G0/G1 cell cycle arrest in ovarian carcinoma cells by the anti-inflammatory drug NS-398, but not by COX-2-specific RNA interference. Oncogene 22: 8653-8661, 2003.

32. Park W, Oh YT, Han JH and Pyo H: Antitumor enhancement of celecoxib, a selective cyclooxygenase- 2 inhibitor, in a Lewis lung carcinoma expressing cyclooxygenase-2. J Exp Clin Cancer Res 27: 66, 2008.

33. Müller-Decker K and Fürstenberger G: The cyclooxygenase-2-mediated prostaglandin signaling is causally related to epithelial carcinogenesis. Mol Carcinog 46: 705-710, 2007.

34. Leahy KM, Ornberg RL, Wang Y, Zweifel BS, Koki AT and Masferrer JL: Cyclooxygenase-2 inhibition by celecoxib reduces proliferation and induces apoptosis in angiogenic endothelial cells in vivo. Cancer Res 62: 625-631, 2002.

35. Mamdani M, Juurlink DN, Lee DS, Rochon PA, Koop A, Naglie G, Austin PC, Laupacis A and Stukel TA: Cyclo-oxygenase-2 inhibitors versus non-selective non-steroidal anti-inflammatory drugs and congestive heart failure outcomes in elderly patients: a population-based cohort study. Lancet 363: 1751-1756, 2004

36. Rasmuson A,KockA,Fuskevăg OM,Kruspig B,Simón-Santamaría J, Gogvadze V, Johnsen JI, Kogner P and Sveinbjörnsson B: Autocrine prostaglandin E2 signaling promotes tumor cell survival and proliferation in childhood neuroblastoma. PLoS One 7: e29331, 2012

37. Pockaj BA, Basu GD, Pathangey LB, Gray RJ, Hernandez JL, Gendler SJ and Mukherjee P: Reduced T-cell and dendritic cell function is related to cyclooxygenase- 2 overexpression and prostaglandin E2 secretion in patients with breast cancer. Ann Surg Oncol 11: 328-339, 2004

38. Iñiguez MA, Rodríguez A, Volpert OV, Fresno M and Redondo JM: Cyclooxygenase-2: a therapeutic target in angiogenesis. Trends Mol Med 9: 73-78, 2003. 